\title{
Evaluation of a Topical Herbal Agent for the Promotion of Bone Healing
}

\author{
Wing-Sum Siu, ${ }^{1,2,3}$ Chun-Hay Ko, ${ }^{1,2}$ Ka-Wing Lam, ${ }^{4}$ Elaine Wat, ${ }^{1,2}$ \\ Wai-Ting Shum, ${ }^{1,2}$ Clara Bik-San Lau, ${ }^{1,2}$ Kam-Ming Ko, ${ }^{5}$ Leung-Kim Hung, \\ David Tai-Wai Lau, ${ }^{1,5}$ and Ping-Chung Leung ${ }^{1,2,3}$ \\ ${ }^{1}$ Institute of Chinese Medicine, 5/F, The CUHK Hong Kong Jockey Club School of Public Health Building, Prince of Wales Hospital, \\ Shatin, New Territories, Hong Kong \\ ${ }^{2}$ State Key Laboratory of Phytochemistry and Plant Resources in West China, The Chinese University of Hong Kong, Shatin, \\ New Territories, Hong Kong \\ ${ }^{3}$ Department of Orthopaedics and Traumatology, The Chinese University of Hong Kong, Shatin, New Territories, Hong Kong \\ ${ }^{4}$ Department of Chemical and Biomolecular Engineering, The Hong Kong University of Science \& Technology, Clear Water Bay, \\ Kowloon, Hong Kong \\ ${ }^{5}$ Division of Life Science, The Hong Kong University of Science \& Technology, Clear Water Bay, Kowloon, Hong Kong
}

Correspondence should be addressed to Ping-Chung Leung; pingcleung@cuhk.edu.hk

Received 23 December 2014; Revised 10 February 2015; Accepted 13 February 2015

Academic Editor: Min Li

Copyright (c) 2015 Wing-Sum Siu et al. This is an open access article distributed under the Creative Commons Attribution License, which permits unrestricted use, distribution, and reproduction in any medium, provided the original work is properly cited.

\begin{abstract}
A topically used Chinese herbal paste, namely, CDNR, was designed to facilitate fracture healing which is usually not addressed in general hospital care. From our in vitro studies, CDNR significantly inhibited the release of nitric oxide from RAW264.7 cells by 51 to $77 \%$. This indicated its anti-inflammatory effect. CDNR also promoted the growth of bone cells by stimulating the proliferation of UMR106 cells up to 18\%. It also increased the biomechanical strength of the healing bone in a drill-hole defect rat model by $16.5 \%$ significantly. This result revealed its in vivo efficacy on facilitation of bone healing. Furthermore, the detection of the chemical markers of CDNR in the skin and muscle of the treatment area demonstrated its transdermal properties. However, CDNR did not affect the bone turnover markers in serum of the rats. With its anti-inflammatory and bone formation properties, CDNR is found effective in promoting bone healing.
\end{abstract}

\section{Introduction}

Fracture is the commonest problem in orthopaedic clinics. The estimated world incidence of adult fractures is around 9.0-22.8 per 1000 people per year [1]. Since fractures are more common among the elderlies, the fracture incidence is expected to further increase in the coming future due to the increasing longevity worldwide [2]. Patients with severe bone fractures are usually hospitalized. The median length of hospital stay after fracture fixation is 13.3 days for men and 19.6 days for women, and the maximum could be over 30 days for hip fractures $[3,4]$. Obviously, fractures reduce the social productivities and intensify the social-economic burden [4$6]$.
Fracture management today is effectively done by surgeons and the healing outcome can be taken for granted. However, the healing process post-op is seldom addressed by hospital and clinical workers yet. The healing of the fracture relies mostly on self-recovery. Patients are usually left unattended except for the pain and inflammation control during hospitalization. In spite of many scientific researches which have been conducted to find the way to promote fracture healing including using biomaterial scaffolds [7-9], growth factors [10, 11], bone morphogenetic proteins [12-14], and biophysical stimulations $[15,16]$, these interventions have not been well accepted for routine clinical applications yet.

Herbal medicine has been used in China for thousands of years and modern researches have demonstrated its 
therapeutic effects on treating various diseases, including cancer [17]. More recently, its applications in modern Western therapy have also been discussed [18]. Facilitation of fracture healing is one of the major areas in traditional Chinese medicine. Traditionally, topical agents have been used to promote the healing of soft tissues and bone fractures. Nonetheless, the formulae of these herbal medicines are too diversified as yet. More importantly, the serious lack of relevant evidence-based scientific support and good systemic documentation of the clinical data makes them not well accepted worldwide.

The aim of this study is to investigate the efficacy of a topical herbal paste on the promotion of bone healing from an evidence-based scientific approach. Both in vitro and in vivo biological platforms will be used to verify the pharmacological properties essential for bone healing, namely, anti-inflammation, proangiogenesis, and cellular regeneration. The most novel component of the study lies in the confirmation of transcutaneous transport of the chemical compounds via the topical application.

Considering that over a hundred of medicinal herbs had been used historically in China for the treatment of skeletal injuries and bone fracture, we select the herbs for the present study according to the classical records and recent scientific papers testifying the pharmacological properties of the herbs related to fracture healing [19].

A herbal paste for topical use (CDNR) was thus created with four herbs, namely, Carthami Flos (C), Dipsaci Radix (D), Notoginseng Rhizoma (N), and Rhei Rhizoma (R). All of them were purchased from a reputable herbal supplier in Hong Kong. The identities of all herbs were authenticated using thin-layer chromatography with procedural references recommended by the Chinese pharmacopoeia [20]. The herbarium voucher specimens of the tested herbs were deposited in the museum bank of the Institute of Chinese Medicine, at the Chinese University of Hong Kong, with reference numbers as follows: Dipsaci Radix: 2010-3279; Rhei Rhizoma: 2010-3280; Notoginseng Rhizoma: 2010-3278; and Carthami Flos: 2010-3335.

\section{Materials and Methods}

2.1. Making of the Topical Agent. Extracts of CDNR were prepared via both aqueous and ethanol extractions. In the aqueous extraction, $250 \mathrm{~g}$ raw materials of each of the four herbs were cut into small pieces $(C: D: N: R$ in $1: 1: 1: 1 \mathrm{w} / \mathrm{w})$ and were soaked in $1.0 \mathrm{~L}$ distilled water for 1 hour. They were boiled twice for 2 hours under reflux and then the aqueous extract was collected and filtered through a piece of absorbent gauze. The residue was further boiled with $95 \%$ ethanol for 2 hours under reflux. The ethanol extract was collected and filtered again. Both of the aqueous and ethanol filtrates were then concentrated at $50^{\circ} \mathrm{C}$ under reduced pressure separately, followed by lyophilizing into powder forms (CDNR(aq) and CDNR(e)) in a freeze dry system (Freezone 12, Labconco, Missouri, USA). The extraction yields of aqueous and ethanol extracts were $39.8 \% \mathrm{w} / \mathrm{w}$ and $5.0 \% \mathrm{w} / \mathrm{w}$, respectively. The paste form of CDNR for topical use was completed by mixing $19.5 \mathrm{~g}$ CDNR(aq) and $3.0 \mathrm{~g} \mathrm{CDNR(e)} \mathrm{with} 17 \mathrm{~mL}$ 50\% ethanol [19].
TABLE 1: Chemical composition of CDNR paste for animal studies.

\begin{tabular}{lcc}
\hline $\begin{array}{l}\text { Herbal } \\
\text { source }\end{array}$ & Name of chemical marker & $\begin{array}{c}\text { Content } \\
\text { (mg/g of paste } \\
\text { mixture powder) }\end{array}$ \\
\hline Carthami & Hydroxysafflor yellow A (HYA) & 2.14 \\
Flos (C) & Kaempferol (KAE) & 0.004 \\
\hline Dipsaci & Asperosaponin VI (ASP) & 19.23 \\
Radix (D) & Oleanolic acid (OA) & 0.05 \\
\hline Notoginseng & Ginsenoside Rg1 (Rg1) & 2.00 \\
Rhizoma (N) & Ginsenoside Rb1 (Rb1) & 5.5 \\
\hline Rhei & Emodin (EMO) & 0.96 \\
Rhizoma (R) & Rhein (RHE) & 1.00
\end{tabular}

2.2. Chemical Composition of Topical Herbal Paste. The chemical composition of the herbal paste was determined by high-performance liquid chromatography-electrospray ionization-mass spectrometry (HPLC-ESI-MS) which included an Agilent 1290 Infinity LC System (Agilent Technologies, Santa Clara, California, USA), equipped with an online degasser, a binary pump, an autosampler, and Agilent 6410 Triple Quad LC/MS, and connected with Agilent MassHunter Workstation software. A Waters HSS T3 $3.5 \mu \mathrm{m}$ $(3.0 \mathrm{~mm} \times 150 \mathrm{~mm})$ HPLC column was used and kept at a temperature of $40^{\circ} \mathrm{C}$. Eight chemical markers and samples were separated using a gradient mobile phase consisting of water (A) and acetonitrile (B). The gradient condition is 0 to $3 \mathrm{~min}, 10-27 \% \mathrm{~B} ; 3$ to $5 \mathrm{~min}, 27-33 \% \mathrm{~B} ; 5$ to $12 \mathrm{~min}, 33-33 \%$ $\mathrm{B} ; 12$ to $13 \mathrm{~min}, 33-80 \% \mathrm{~B} ; 13$ to $16 \mathrm{~min}, 80-90 \% \mathrm{~B} ; 16$ to $20 \mathrm{~min}, 90-90 \% \mathrm{~B}$. The flow rate was set at $0.5 \mathrm{~mL} / \mathrm{min}$ and the injection volume was $20 \mu \mathrm{L}$.

MS analysis was performed on Agilent 6410 Triple-Quad LC/MS equipped with an ESI source [21] and monitored in negative ion mode and multiple reaction monitoring mode using target ions at $\mathrm{m} / z \quad 611.2 \rightarrow 325.0$ for hydroxysafflor yellow A (HYA); $m / z 285.0 \rightarrow 117.0$ for kaempferol (KAE); $m / z$ $927.5 \rightarrow 603.3$ for asperosaponin VI (ASP); $m / z 455.3 \rightarrow 407.4$ for oleanolic acid (OA); $m / z 799.5 \rightarrow 637.4$ for ginsenoside $\operatorname{Rg} 1$ (Rg1); $m / z 1107.6 \rightarrow 119.0$ for ginsenoside Rb1 (Rb1); $m / z$ $269.0 \rightarrow 241.0$ for emodin (EMO), and $m / z 283.0 \rightarrow 239.0$ for rhein (RHE). The abundance of the markers was determined quantitatively (Table 1).

All the chemicals were purchased from Sigma-Aldrich (St. Louis, Missouri, USA). Both methanol and acetonitrile were HPLC-grade (CHROMASOLV Plus, $\geq 99.9 \%$ ). All the chemical markers were purchased from Tauto Biotech Co., Ltd (Shanghai, China). Their purities were over 97\%.

2.3. In Vitro Studies. In vitro studies were targeting antiinflammation and cellular proliferation.

2.3.1. Cell Cultures. Murine monocyte/macrophage RAW264.7 and rat osteosarcoma UMR-106 cells were purchased from American Type Culture Collection (Manassas, VA, USA). RAW264.7 and UMR-106 cells were maintained in high-glucose DMEM (d-glucose: $3500 \mathrm{mg} / \mathrm{L}$; GIBCO, USA), $10 \%$ v/v fetal bovine serum (FBS; Gibco, Gran Island, NY, USA), and $1 \%$ penicillin-streptomycin (PS; Gibco, Gran 
Island, NY, USA). All cells were maintained at $37^{\circ} \mathrm{C}, 5 \% \mathrm{CO}_{2}$ humidified incubator.

(i) Nitric Oxide (NO) Inhibitory Assay. Macrophage cells RAW264.7 $\left(4 \times 10^{5}\right.$ per well) were seeded. With $0.1 \mu \mathrm{g}$ of lipopolysaccharide (LPS) per mL of medium, CDNR(aq) or CDNR(e) was added (at concentrations ranging from 0 to $400 \mu \mathrm{g} / \mathrm{mL}$ ) and incubated for $24 \mathrm{~h}$. The nitrite accumulation in the supernatant was determined by Griess reagent (Sigma, St. Louis, MO, USA) [22, 23] read at a wavelength of $540 \mathrm{~nm}$. Cell viability of RAW264.7 in various concentrations of $\operatorname{CDNR}(\mathrm{aq})$ or $\operatorname{CDNR}(\mathrm{e})$ was determined by MTT assay (3-[4,5-dimethylthiazol-2-yl]-2,5-diphenyltetrazolium bromide) (Sigma, St. Louis, MO, USA) [22]. The relative amount of viable cells was determined using optical density at $540 \mathrm{~nm}$ and expressed as the percentage of Control samples without treatment.

(ii) Determination of Production of Proinflammatory Cytokines Using ELISA Assay. The RAW264.7 cell culture supernatants were subjected to test for the concentrations of cytokines, TNF- $\alpha$, IL- 6 , and IL-1 $\beta$, by enzyme-linked immunosorbent assay (ELISA) [22]. These assays were carried out according to the procedures recommended in the ELISA kit manual (BD Biosciences, San Jose, CA, USA).

(iii) Osteoblast Proliferation and Viability Assays. The osteoblast proliferation and viability were measured using 5bromo-2' -deoxyuridine (BrdU) [24] incorporation and MTT cell viability assay $[19,25]$, respectively. Briefly, UMR-106 cells were seeded in DMEM with 10\% v/v FBS. After $24 \mathrm{~h}$, cells were exposed to different concentrations of $\operatorname{CDNR}(\mathrm{aq})$ or CDNR(e) in DMEM with 10\% v/v FBS. An ELISA kit (Roche, Palo Alto, CA, USA) was used to determine the proliferation of the osteoblast in the BrdU incorporation assay. For the MTT assay, MTT solution $(5 \mathrm{mg} / \mathrm{mL})$ was added directly to the medium in each well, and the plate was then incubated at $37^{\circ} \mathrm{C}$ for $4 \mathrm{~h}$. All medium was then aspirated and replaced with dimethyl sulfoxide (DMSO, Sigma-Aldrich, USA). The relative amount of viable cells was determined at optical density at $540 \mathrm{~nm}$. The osteoblast proliferation and cell viability were expressed as the percentage of Control samples without treatment.

2.4. In Vivo Study. The animal model (rat) with artificially created bone defects via cortical and metaphyseal drillings is a recognized model for the study of bone healing $[26,27]$.

2.4.1. Animals Models with Bone Injury. Twenty female Sprague-Dawley rats, aged $15.2 \pm 1.41$ months (mean \pm standard deviation), were obtained from the Laboratory Animal Service Centre of the Chinese University of Hong Kong (CUHK). All of them were housed in a temperaturecontrolled $\left(25^{\circ} \mathrm{C}\right)$ and light-controlled $(12 \mathrm{~h}$ light/dark cycle) environment. All the animals were allowed access to standard rodent chow and water ad libitum throughout the study. After seven days of acclimatization, bone defects in the form of larger drill holes were created in the femur and tibia of the rats. The animal study had been approved by the Animal Experimental Ethics Committee of CUHK.

The rat was firstly anesthetized using a cocktail of ketamine and xylazine intramuscularly. On the left femur, two side-by-side drill holes ( $2 \mathrm{~mm}$ in diameter each) were created using an electric drill on the midshaft of the femur through an anterior-posterior approach. The two holes were connected to form a $2 \mathrm{~mm} \times 4 \mathrm{~mm}$ defect using a dental milling burr. On the right tibia, (opposite leg) a bone defect, $2.4 \mathrm{~mm}$ in diameter, was made using the electric drill at the proximal metaphysis via a medial-lateral approach. All the drilled holes were cleaned thoroughly, irrigating with $0.9 \%$ sterile saline, making sure that small bone fragments would not remain.

The rats were divided into two groups with 10 rats each. In the control group (Control), the left femur and the right tibia were covered with just thin self-adhesive films without any treatment. In the herbal paste treatment group (CDNR), $0.5 \mathrm{~mL}$ CDNR paste was applied topically upon the left femur and right tibia, covering the operated sites. The paste was protected from falling off and drying with thin self-adhesive films. All the films and topical treatment paste were renewed at 2-day intervals. The whole treatment period lasted for 6 weeks.

\subsubsection{Assessments}

(i) Change in Microarchitecture Using Microcomputed Tomography (Micro-CT). In vivo longitudinal changes in bone volume (BV) of the drill-hole bone defect at the proximal tibia of the rat were monitored using micro-CT (MicroCT 40, Scanco Medical, Bassersdorf, Switzerland) within the experimental period. The rat was anesthetized using a cocktail of ketamine and xylazine intramuscularly and then fixed on a plastic holder to ensure a repeatable positioning. The right proximal tibia was scanned for 700 slices with energy $70 \mathrm{kVp}$ and intensity $114 \mu \mathrm{A}$. The isotropic resolution was $12.5 \mu \mathrm{m}$ per voxel. The volume of interest was determined within 250 continuous slices covering the whole drill hole. Segmentation parameters to define BV were fixed at sigma $=0.5$, support $=1.0$, and threshold $=245$ for all analyses. These in vivo measurements were performed one day before the surgical drill-hole operation (Day -1), right after the operation (Day 0 ), and on 14, 28, and 42 days post-op (Day 14, Day 28, and Day 42 , resp.).

(ii) Bone Turnover Monitoring by Measuring Biochemical Markers. Prior to microarchitecture measurement using micro-CT, blood was collected from the orbital venous plexus after the rat was anesthetized. Sera were obtained after centrifugation at $3000 \mathrm{rpm}$ for 15 minutes at $4^{\circ} \mathrm{C}$. They were then aliquoted and stored at $-80^{\circ} \mathrm{C}$ before the biochemical markers were measured. Bone-specific alkaline phosphatase (BALP) was measured following the wheat germ lectin (WGL) (Sigma, St. Louis, MO, USA) precipitation method $[28,29]$. Deoxypyridinoline (Dpd) was also measured using commercial available ELISA kit (MyBioSource, San Diego, USA). 
(iii) Biomechanical Bending Tests for Bone Strength Assessment. The rats were euthanized on Day 42. Their left and right femora were harvested. Excessive soft tissue was removed but the periosteum was preserved. Four-point bending tests were performed using a Hounsfield material testing machine (KM25, Redhill, United Kingdom). A load cell with maximum $2500 \mathrm{~N}$ was mounted. The spans of the upper and lower supports were 8.0 and $20 \mathrm{~mm}$, respectively. The drill-hole bone defect at the midshaft of the femur was located in the middle of the two upper supports and then the whole femur was loaded at a constant speed of $5 \mathrm{~mm} / \mathrm{min}$ in posterioranterior approach until failure. Load and work done at yield were recorded for analysis. All the data of the drilled femur (left) were normalized with the normal femur (right). The results were expressed as the normalized percentage based on the normal femur.

\subsection{Testing for Transcutaneous Transport of the Topical Agent.} In order to study whether the topical herbal materials go across the skin barrier, the presence and quantity of essential herbal chemical markers of the medicinal herbs used (CDNR) in the rat skin and underneath muscle were measured. $0.1 \mathrm{~g}$ of rat skin and muscle was collected from the region of the topical paste application after the rat was sacrificed. The tissues were rinsed with PBS gently and then homogenized with $1 \mathrm{~mL}$ of homogenization buffer $(50 \mathrm{mM}$ Tris, $1 \mathrm{mM}$ DTT, and $\mathrm{pH}$ 7.5) using a homogenizer on ice. Then, $1 \mathrm{~mL}$ of acetonitrile was added to the homogenate and filtered for analysis. The presence of the chemical markers of the herbal compounds was revealed using the LC-MS equipment. The abundance of each marker was quantitatively determined.

2.6. Statistical Analysis. For the multiple group comparisons of cell proliferation, viability, NO, and cytokine production, the significance of the differences among the treatment groups and their respective control groups were tested by one-way ANOVA with Dunnett's post hoc test. For the in vivo studies, the differences between Control and the treatment group (CDNR) were analyzed with nonparametric MannWhitney $U$ test. The results of bone microarchitecture (from micro-CT) and biochemical markers (BALP and Dpd) were analyzed following repeated measure ANOVA. Data were presented as mean \pm standard deviation (SD) unless otherwise specified. All the statistical analyses were carried out using GraphPad Prism version 5.0 for Windows (GraphPad Software, San Diego, CA, USA) and tests were two-sided, with $P<0.05$ being considered as statistical significant.

\section{Results}

3.1. In Vitro Anti-Inflammatory Effects of CDNR. Results demonstrated that $\operatorname{CDNR}(\mathrm{aq})$ did not affect the NO production in LPS-induced RAW264.7 cells (Figure 1(a)). In contrast, CDNR(e), at $100 \mu \mathrm{g} / \mathrm{mL}$ and $200 \mu \mathrm{g} / \mathrm{mL}$, significantly suppressed the NO production in RAW264.7 cells after LPS induction by $51 \%$ and $77 \%$, respectively $(P<$ 0.001 ), when compared to the control group without herbal
TABLE 2: Change of bone volume of the drill-hole bone defect.

\begin{tabular}{lccc}
\hline & Control $\left(\mathrm{mm}^{3}\right)$ & CDNR $\left(\mathrm{mm}^{3}\right)$ & $P$ value \\
\hline Day -1 & $20.223 \pm 1.589$ & $19.509 \pm 2.111$ & 0.457 \\
Day 0 & $15.045 \pm 1.696$ & $15.427 \pm 2.247$ & 0.707 \\
Day 14 & $18.249 \pm 1.766$ & $18.091 \pm 2.413$ & 0.883 \\
Day 28 & $17.582 \pm 1.801$ & $17.231 \pm 2.180$ & 0.730 \\
Day 42 & $18.020 \pm 1.949$ & $17.111 \pm 1.826$ & 0.352 \\
\hline
\end{tabular}

There was no significant difference in the bone volume $\left(\mathrm{mm}^{3}\right)$ of the drillhole bone defect in tibial metaphysis measured by using micro-CT between Control and CDNR at each time point. Day -1 and Day 0: measurement prior to and right after the drill-hole bone defect creation, respectively. Data are expressed as mean \pm standard deviation.

treatment $(0 \mu \mathrm{g} / \mathrm{mL})$ (Figure $1(\mathrm{~b}))$. However, CDNR(e) was nontoxic to the cells because it did not affect the normal cellular activity at concentration lower than $200 \mu \mathrm{g} / \mathrm{mL}$ (data not shown). CDNR(e) also suppressed the proinflammatory cytokines production in RAW264.7 cells after LPS induction. At $200 \mu \mathrm{g} / \mathrm{mL}$, it effectively downregulated the production of TNF- $\alpha$ (by $25.2 \%, P<0.05$; Figure 1(c)), IL-6 (by $35.2 \%, P<0.001$; Figure $1(\mathrm{~d})$ ), and IL-1 $\beta$ (by $20.9 \%, P<$ 0.05; Figure $1(\mathrm{e})$ ) when compared with its corresponding controls, which further indicated that $\operatorname{CDNR}(\mathrm{e})$ was antiinflammatory.

3.2. In Vitro Osteogenic Effect of CDNR. As shown in Figure 2(a), after treatment of CDNR(aq) for $24 \mathrm{~h}$, significant proliferative effects were observed in UMR-106 cells from 25 to $100 \mu \mathrm{g} / \mathrm{mL}(P<0.001)$ with increments of $10.8 \%$ to $22 \%$. Similar significant cell viability-enhancement effects were observed in UMR-106 from 6.25 to $100 \mu \mathrm{g} / \mathrm{mL}$ (Figure 2(b)). CDNR(e) did not affect UMR-106 cells after $24 \mathrm{~h}$ of treatment and up to $100 \mu \mathrm{g} / \mathrm{mL}$ in both assays.

\subsection{In Vivo Effect of CDNR on the Microarchitecture of Drilled} Bone. From the quantitative micro-CT analysis at the drillhole bone defects over the proximal tibial metaphyses of the rats, bone volume (BV) (from Day 0 to Day 42) was changed significantly $(P<0.001)$ depending on the time (number of days post-op) in both Control and CDNR groups. It changed from $15.43 \mathrm{~mm}^{3}$ to $17.11 \mathrm{~mm}^{3}$ in CDNR while from $15.05 \mathrm{~mm}^{3}$ to $18.02 \mathrm{~mm}^{3}$ in Control (Table 2). However, there was no significant difference in the BV between the CDNR and the control group when it was compared at each specific time point throughout the study. Since the femur was designed for biomechanical tests, no micro-CT data was obtained from the femur.

3.4. In Vivo Effects of Topical CDNR Application on Systemic Bone Remodeling. There was a significant overall increase in the bone-specific alkaline phosphatase (BALP) level in serum in both control and CDNR groups of the rats after the drillhole bone defect had been created $(P<0.001)$. However, there was no significant difference between the two groups at each time point throughout the study (Table 3 ). On the other hand, an overall significant descending trend of serum deoxypyridinoline (Dpd) was observed after the surgery in 


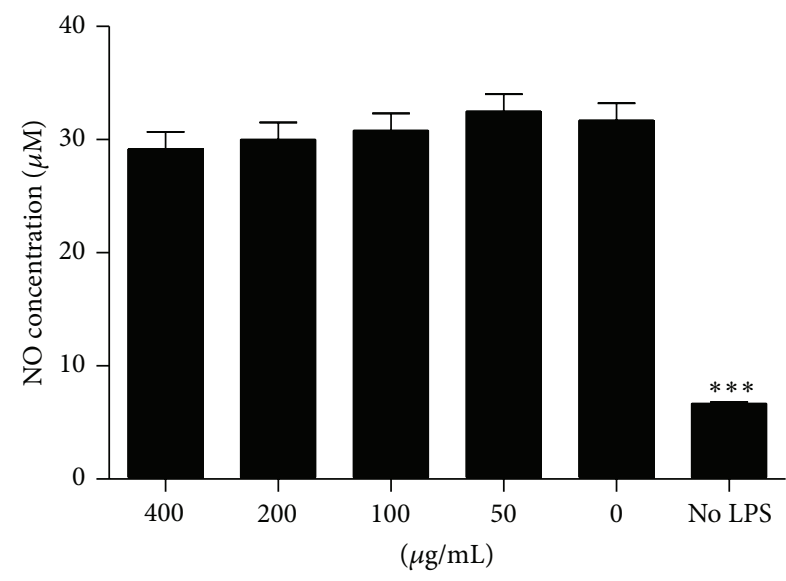

(a)

TNF- $\alpha$

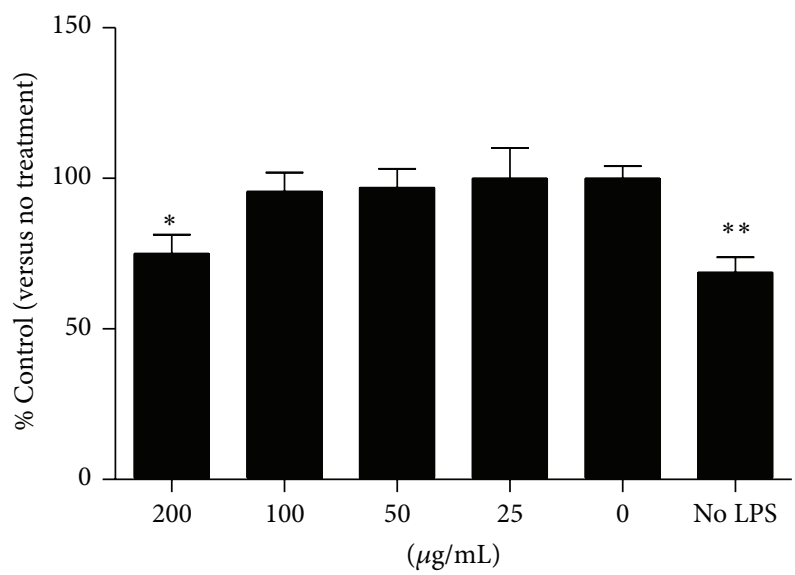

(c)

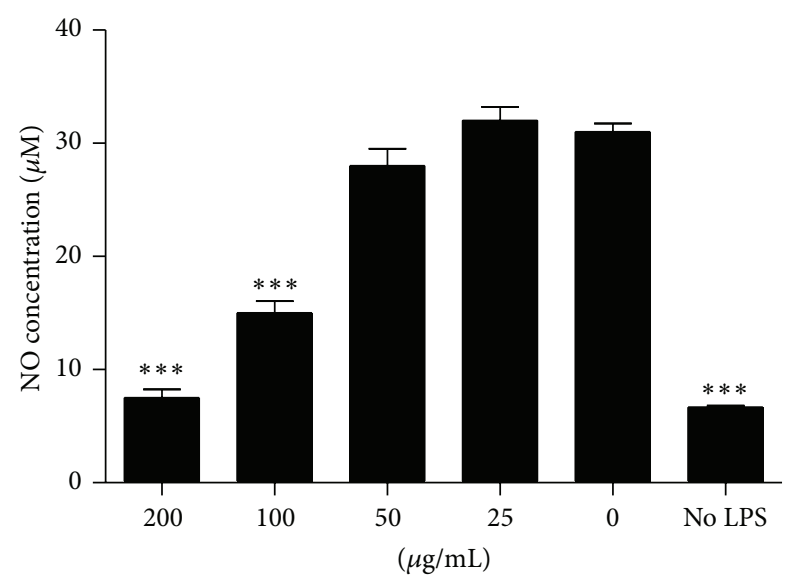

(b)

IL-6

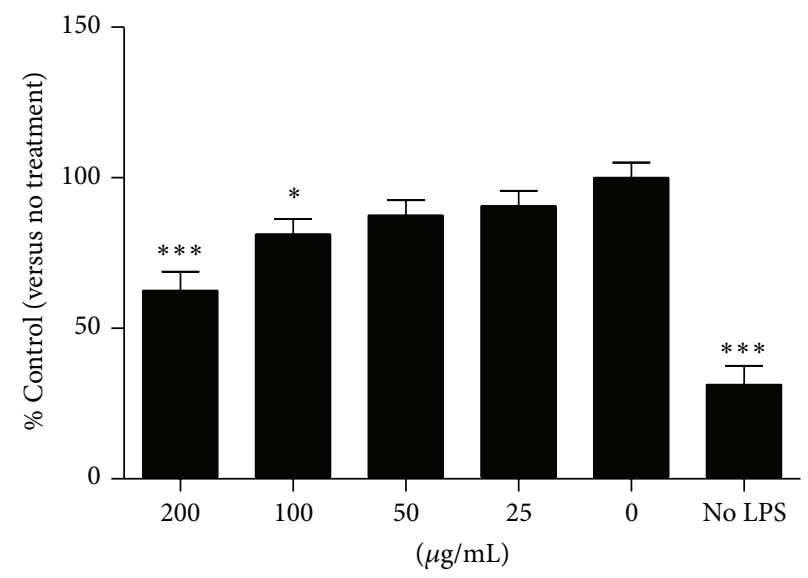

(d)

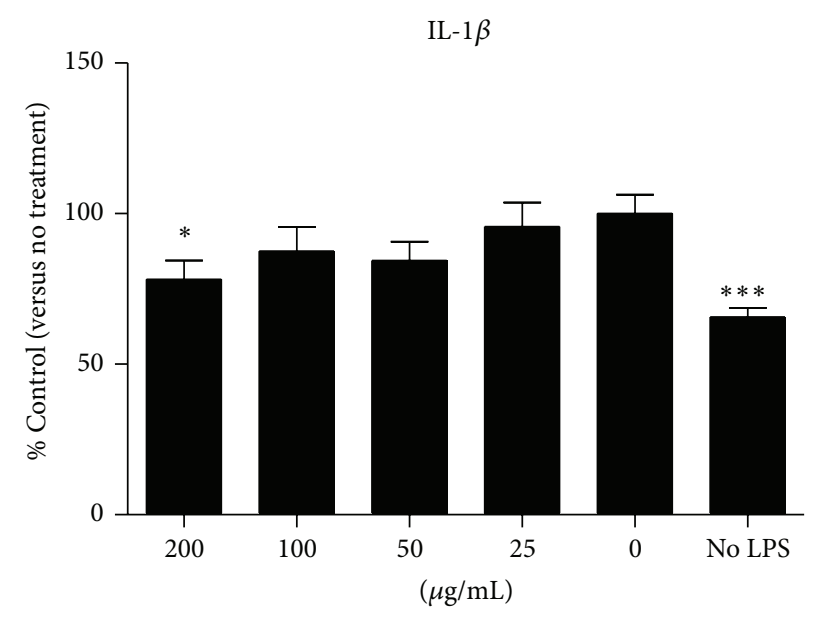

(e)

Figure 1: Anti-inflammatory effects of CDNR. (a, b) Effect of aqueous (CDNR(aq)) and ethanol (CDNR(e)) component of CDNR on nitric oxide (NO) production by RAW264.7 induced by LPS; (c, d, and e) Effect of CDNR(e) on TNF- $\alpha$, IL-6, and IL-1 $\beta$ production by RAW264.7. Data are expressed as mean and standard deviation (error bar). ${ }^{*} P<0.05,{ }^{* *} P<0.01$, and ${ }^{* * *} P<0.001$ versus Control $(0 \mu \mathrm{g} / \mathrm{mL})$. 


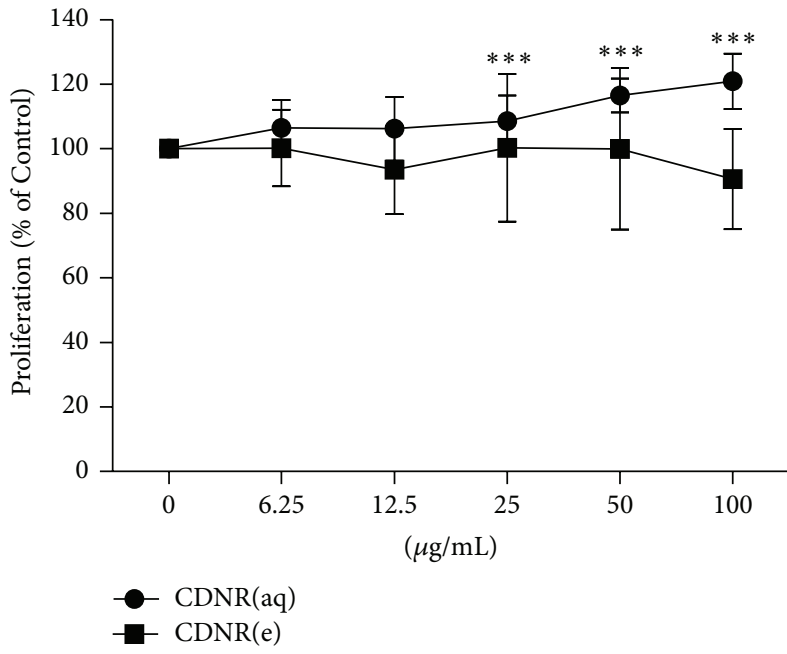

(a)

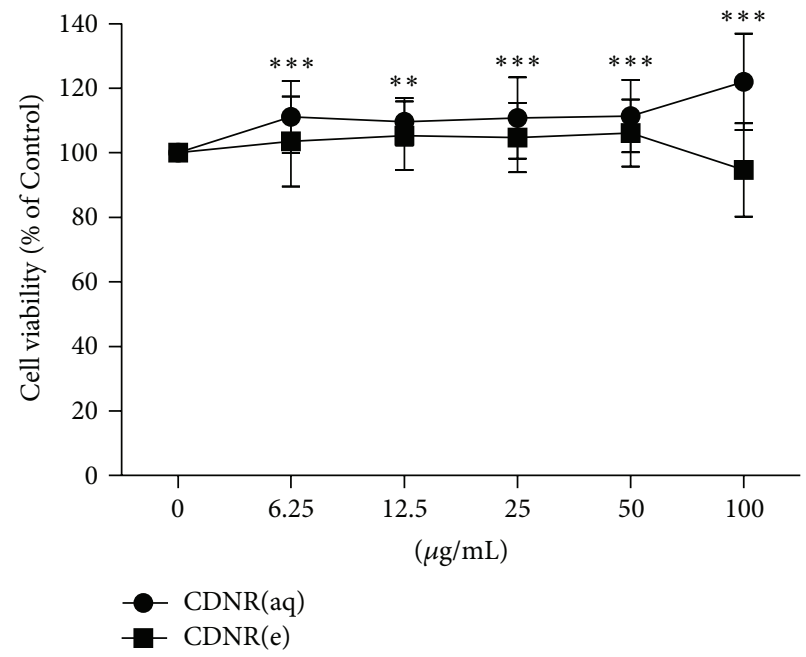

(b)

FIGURE 2: Osteogenic effect of CDNR on bone cells. (a) UMR106 proliferation at different concentration of CDNR assessed by BrdU assay; (b) UMR106 viability at different concentration of CDNR assessed by MTT assay. (CDNR(aq), CDNR(e)) Aqueous and ethanol component of CDNR. Data are expressed as mean and standard deviation (error bar). ${ }^{* *} P<0.01,{ }^{* * *} P<0.001$ versus Control $(0 \mu \mathrm{g} / \mathrm{mL})$.

TABLE 3: Change of biochemical markers in serum after drill-hole bone defects had been created.

\begin{tabular}{|c|c|c|c|c|c|c|}
\hline & \multicolumn{3}{|c|}{ BALP (U/L) } & \multicolumn{3}{|c|}{ Dpd (nmol/L) } \\
\hline & Control & CDNR & $P$ value & Control & CDNR & $P$ value \\
\hline Day -1 & $3.582 \pm 1.207$ & $3.473 \pm 1.391$ & 0.882 & $3.665 \pm 1.421$ & $2.862 \pm 0.577$ & 0.229 \\
\hline Day 14 & $4.887 \pm 1.610$ & $4.606 \pm 1.649$ & 0.762 & $2.964 \pm 1.118$ & $2.614 \pm 0.710$ & 0.532 \\
\hline Day 28 & $5.909 \pm 2.235$ & $5.120 \pm 2.458$ & 0.557 & $2.761 \pm 1.657$ & $2.303 \pm 0.624$ & 0.540 \\
\hline Day 42 & $4.991 \pm 1.605$ & $5.283 \pm 2.153$ & 0.785 & $2.791 \pm 1.586$ & $1.891 \pm 0.411$ & 0.208 \\
\hline
\end{tabular}

There was no significant difference in the serum biochemical markers between Control and CDNR at each time point. Day -1: blood was collected prior to the drill-hole bone defect creation; BALP: bone-specific alkaline phosphatase; Dpd: deoxypyridinoline. Data are expressed as mean \pm standard deviation.

both groups $(P=0.003)$. Similar to BALP, no difference of Dpd between the two groups at each time point was found neither (Table 3).

3.5. In Vivo Effect of CDNR on the Bone Strength (Biomechanical Properties) after Healing. Femora with cortical drill holes showed $16.5 \%$ higher normalized yield strength (\% from the contralateral normal femur) in the CDNR treated group when compared with the Control $(P<0.05)$ after 42 days of treatment (Figure 3(a)). Similar findings were observed when the total work done at yield point was analyzed. CDNR treated bone showed $13.8 \%$ higher energy than the Control to reach the yield point $(P<0.05)$ (Figure 3(b)). The 4-point bending tests illustrated that the biomechanical properties of the healing at the drill-hole bone defects were improved significantly when the topical herbal paste was applied.

3.6. Transdermal Transport of the Herbal Compounds through the Rat Skin and Muscle. The transdermal transport of CDNR was determined by identifying the relevant chemical marker compounds in the skin and muscle after 6 weeks of herbal treatment using Q-TOF LC/MS technique. As shown in Figure 4(a), all the chemical markers of CDNR, except oleanolic acid (OA), were detected in the rat skin with asperosaponin VI (ASP) showing the most abundance. Similarly, those 7 chemical markers detected in the skin, except kaempferol (KAE), could also be found in the muscle. $\mathrm{OA}$ was nondetectable neither in the muscle. With a little bit difference from skin, rhein (RHE) but not the ASP was found to be the most abundant in the muscle (Figure 4(b)).

\section{Discussion}

Scientific studies on topical TCM treatment for fracture healing are seldom reported in international journals. As a result, there is lack of scientific evidence to support the use of topical herbal paste to facilitate fracture healing clinically. This study is one of the few scientific reports exploring the pharmacological activities of a topical agent from two standard directions, namely, direct cellular influences using standard cell lines for the study of anti-inflammation and cellular proliferation (in vitro) and life-animal (in vivo) situation of bone regeneration.

In the in vitro studies, the aqueous component of CDNR promoted the UMR-106 proliferation significantly. 


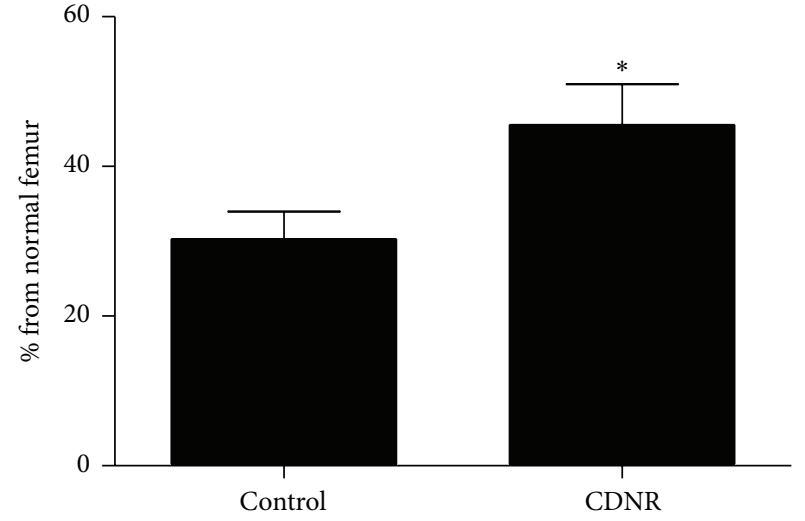

(a)

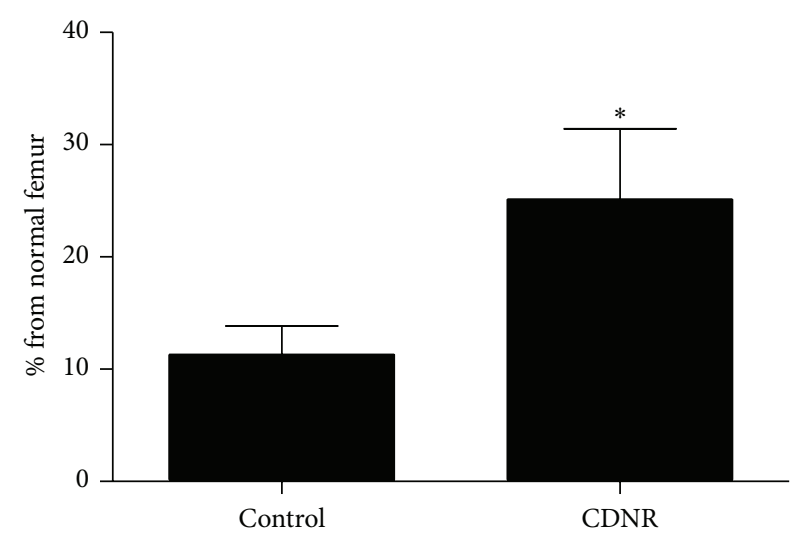

(b)

Figure 3: Biomechanical properties of femur with drill-hole bone defect. (a) Yield strength; (b) work done at yield strength. Data are expressed as mean and standard error of mean (error bar). ${ }^{*} P<0.05$ versus Control.

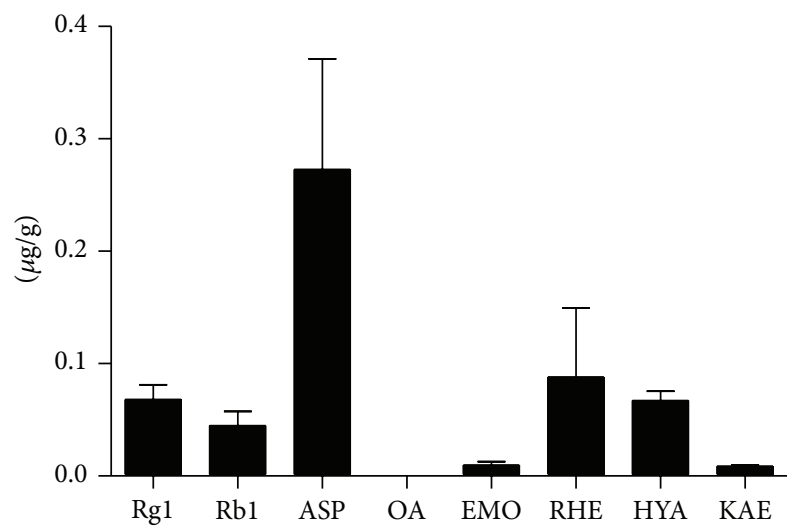

(a)

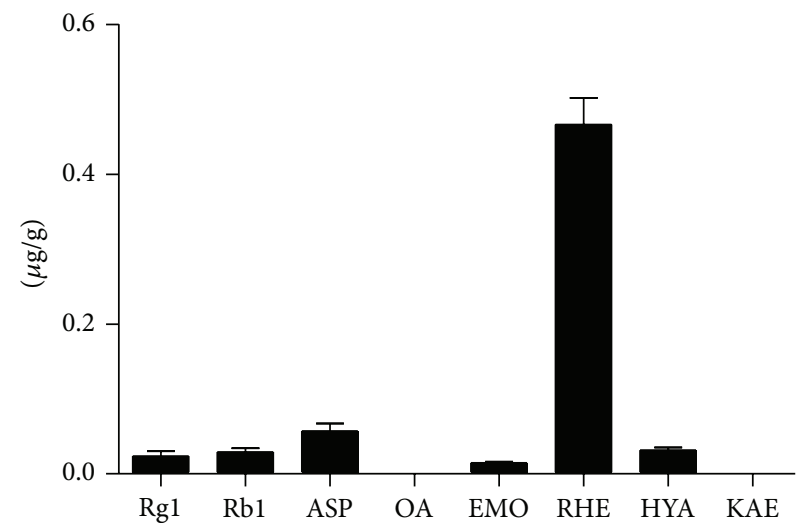

(b)

FIGURE 4: Concentration of the chemical markers of CDNR remained in the soft tissues of the rat. (a) Skin; (b) muscle. Data are expressed as mean and standard deviation (error bar). Rg1: ginsenoside Rg1; Rb1: ginsenoside Rb1; ASP: asperosaponin VI; OA: oleanolic acid; EMO; emodin; RHE: rhein; HYA: hydroxysafflor yellow A; KAE: kaempferol.

This observation indicated that CDNR could promote osteogenesis which must be an important mechanism during bone repair, particularly during the reparative phase of fracture healing when endochondral ossification takes places and osteoblasts start to form new lamellar bone on the cartilaginous callus [30].

Anti-inflammation is one of the key principles in the treatment of fractures as well as traditional bone setters' advocations. It controls the swelling and relieves the pain from the fracture site and the surrounding soft tissue which promotes overall healing. During inflammation, there is an increased production of various mediators such as arachidonic acid metabolites and cytokines. Besides, there are confirming evidences stating that nitric oxide (NO) produced by inducible NO synthase (iNOS) plays one of the important roles in inflammatory disorders such as rheumatoid arthritis $[31,32]$. Therefore, chemicals that inhibit the NO production by iNOS in macrophages would be the potential treatments to reduce inflammatory responses [22]. In this regard, it is meaningful to examine the effects of CDNR on NO production. One of our in vitro studies demonstrated that the ethanol component of CDNR suppressed LPS-induced nitric oxide and cytokines production significantly. These observations indicated that there are active ingredients in CDNR that can suppress inflammation and thus controls the swelling and relieves the pain during fracture healing.

The ultimate goal of bone repair is to restore the bone strength of the injured bone to its original level without fracture or defect [33]. Assessment on the bone strength during fracture or defect healing in preclinical studies is essential to evaluate the effectiveness of an intervention on bone repair. The biomechanical tests of the current study showed strong evidences of healing promotion from the topical use of the herbal paste. The higher bending yield strength and work load ability of the femur in the CDNR group than in the control group revealed that the treatment elevated the strength of the bone that can tolerate much more stress and energy before permanent deformation which 
could be caused by microfractures. Perhaps the topical use of CDNR may reduce the risk of refracture during fracture healing due to insufficient structural remodeling [34]. CDNR, therefore, might act like some antiresorptive pharmaceutical agents when they help building up bone density and bone strength in the prevention of refracture [35].

Nowadays, microstructure of the trabecular bone is one of the most important parameters in orthopaedic researches. The microarchitecture of trabecular bone was highly correlated with bone strength $[36,37]$. Therefore, assessment on microarchitecture gives a new dimension to researchers in terms of clinical observations of bone. In the micro-CT analysis of the current study, no difference in BV in the trabecular region of the tibia was found between CDNR and Control. It might be due to the fact that the bone healing process in trabecular metaphysis is different from that in cortical diaphysis. Healing of bone lesions involves many factors, including different processes according to the type of bone (cortical or cancellous), position and size of the wound, and the biomechanical environment provided onto the injured bone [38]. Diaphyseal defect on femur of mice was rapidly repaired while the epimetaphyseal defect failed to bridge the cortical gap even 13 weeks after surgery [39]. Another study reported that when the defect with the same size was located at the metaphysis of distal femur of mice, it bridged completely within 6 weeks [40].

Bone formation and bone resorption are two tightly coupled activities in a continuous and dynamic process of bone remodeling. During bone resorption, calcium and other matrix constituents are released into the bloodstream. When in bone formation, skeletal-specific proteins (enzymes or matrix components) can leach into the circulation. These biochemical markers can be measured by a noninvasive, systemic, and sensitive method to detect changes of bone turnover in a short interval of time [41]. These measurements have also been used to predict bone fracture $[42,43]$. The bone formation marker BALP increased in both groups of rats in this study. This observation illustrated that the osteoblastic activity of the rats, whether Control or CDNR, was increased after the drill-hole bone defect had been created. The data was coherent with the results of a previous study on human, in which the increase of BALP started to be observed from the 7 th day after fracture, elevated by nearly $200 \%$ to week 12 , and was maintained at high level thereafter [44]. In the fracture of the tibial shaft, BALP increased at 4 weeks [45]. On the other hand, the serum concentration of the bone resorption marker Dpd was found reduced in both groups of rats postop. It indicated that bone resorption activity was retarded during bone repair. Similar findings were also reported in the studies using bovine [46] and canine [47] model of bone fracture healing. They showed that the serum TRAP activity, another bone resorption biochemical marker, was low during the postfracture period. On the whole, there was a net bone gain after the systemic bone remodeling process of the rats in the processes of drill-hole bone repair. Apparently, the topical CDNR treatment did not alter this process systematically. Its promotional effect on bone healing could possibly be a local response.
The essential factor required for the efficacy of any topical treatment is its transdermal transport. The efficiency of the transport of the active ingredients of the drug depends on the physicochemical properties of the molecules, including the size (molecular weight), charge, and hydrophilicity (water solubility) [48-50]. In the transdermal experiment of this study, almost all the chemical markers of CDNR were detected by LC/MS in both the skin and muscle at the treatment sites of the rats. This observation illustrated that each herbal component of CDNR did pass through the skin barrier to cast its therapeutic effect. It further supports the hypothesis that the herbal components of CDNR influence bone healing via local therapeutic responses.

Topical agents have been popular before the present era of fracture management. Now that fracture treatment is well developed, clinicians have isolated themselves from the traditional treatment applications. As a matter of fact, since no scientific records are available neither with the biological activities nor with the more basic transdermal transport, the old practice might not deserve much attention.

However, since topical herbal agents are still widely used in the households and there might be special indications of utilization, it would be necessary to start serious scientific explorations. Indeed, some difficult fracture healings might still benefit from simple, harmless topical applications as long as toxicity and sensitivity could be ruled out. Moreover, the anti-inflammatory effects could also help with the recovering of soft tissue injuries [51].

\section{Conclusions}

The present study demonstrated for the first time that a 4-herb formula (CDNR) was effective in the control of inflammation and facilitation of bone regeneration when used topically. Its efficacy on the promotion of cortical bone repair was also revealed in the rat experiments. This study gave us sufficient scientific evidences that our novel herbal paste is a safe and promising supplement agent to facilitate bone healing under special circumstances.

\section{Conflict of Interests}

The authors declare that there is no conflict of interests regarding the publication of this paper.

\section{Authors' Contribution}

Wing-Sum Siu and Chun-Hay Ko equally contributed to the paper.

\section{Acknowledgment}

This work was supported by Research Fund for Innovation and Technology Support Programme (ITP/014/10NP), Innovation and Technology Commission, Hong Kong. 


\section{References}

[1] C. M. Court-Brown and B. Caesar, "Epidemiology of adult fractures: a review," Injury, vol. 37, no. 8, pp. 691-697, 2006.

[2] O. Johnell, C. Cooper, S. Cummings, C. Slemenda, and E. Seeman, "The socioeconomic burden of fractures: today and in the 21st century," The American Journal of Medicine, vol. 103, supplement 2, pp. 20S-25S, 1997.

[3] R. S. Morrison, J. Magaziner, M. A. McLaughlin et al., "The impact of post-operative pain on outcomes following hip fracture," Pain, vol. 103, no. 3, pp. 303-311, 2003.

[4] K. Lippuner, J. von Overbeck, R. Perrelet, H. Bosshard, and P. Jaeger, "Incidence and direct medical costs of hospitalizations due to osteoporotic fractures in Switzerland," Osteoporosis International, vol. 7, no. 5, pp. 414-425, 1997.

[5] M. L. Kilgore, M. A. Morrisey, D. J. Becker et al., "Health care expenditures associated with skeletal fractures among medicare beneficiaries, 1999-2005," Journal of Bone and Mineral Research, vol. 24, no. 12, pp. 2050-2055, 2009.

[6] National Osteoporosis Society: 25th anniversary report-a fragile future, 2011, http://www.nos.org.uk/document.doc?id= 904.

[7] D. J. Hak, "The use of osteoconductive bone graft substitutes in orthopaedic trauma," Journal of the American Academy of Orthopaedic Surgeons, vol. 15, no. 9, pp. 525-536, 2007.

[8] G. M. Calori, E. Mazza, M. Colombo, and C. Ripamonti, “The use of bone-graft substitutes in large bone defects: any specific needs?” Injury, vol. 42, supplement 2, pp. S56-S63, 2011.

[9] K. A. Hing, L. F. Wilson, and T. Buckland, "Comparative performance of three ceramic bone graft substitutes," The Spine Journal, vol. 7, no. 4, pp. 475-490, 2007.

[10] A. Lode, C. Wolf-Brandstetter, A. Reinstorf et al., "Calcium phosphate bone cements, functionalized with VEGF: release kinetics and biological activity," Journal of Biomedical Materials Research Part A, vol. 81, no. 2, pp. 474-483, 2007.

[11] E. Wernike, M.-O. Montjovent, Y. Liu et al., "VEGF incorporated into calcium phosphate ceramics promotes vascularisation and bone formation in vivo," European Cells and Materials, vol. 19, pp. 30-40, 2010.

[12] J. C. Y. Cheng, X. Guo, L. P. Law, K. M. Lee, D. H. K. Chow, and R. Rosier, "How does recombinant human bone morphogenetic protein-4 enhance posterior spinal fusion?” Spine, vol. 27 , no. 5 , pp. 467-474, 2002.

[13] G. Schmidmaier, R. Capanna, B. Wildemann, T. Beque, and D. Lowenberg, "Bone morphogenetic proteins in critical-size bone defects: what are the options?” Injury, vol. 40, supplement 3, pp. S39-S43, 2009.

[14] N. Mokbel, N. Naaman, J. Nohra, and N. Badawi, "Healing patterns of critical size bony defects in rats after grafting with bone substitutes soaked in recombinant human bone morphogenetic protein-2: histological and histometric evaluation," British Journal of Oral and Maxillofacial Surgery, vol. 51, no. 6, pp. 545-549, 2013.

[15] O. Akkus, F. Korkusuz, S. Akin, and N. Akkas, "Relation between mechanical stiffness and vibration transmission of fracture callus: an experimental study on rabbit tibia," Proceedings of the Institution of Mechanical Engineers, Part H, vol. 212, no. 5, pp. 327-336, 1998.

[16] K. Shakouri, B. Eftekharsadat, M. R. Oskuie et al., "Effect of lowintensity pulsed ultrasound on fracture callus mineral density and flexural strength in rabbit tibial fresh fracture," Journal of Orthopaedic Science, vol. 15, no. 2, pp. 240-244, 2010.
[17] C.-Q. Ling, X.-Q. Yue, and C. Ling, "Three advantages of using traditional Chinese medicine to prevent and treat tumor," Journal of Integrative Medicine, vol. 12, no. 4, pp. 331-335, 2014.

[18] C. Q. Ling, L. N. Wang, Y. Wang et al., "The roles of traditional Chinese medicine in gene therapy," Journal of Integrative Medicine, vol. 12, no. 2, pp. 67-75, 2014.

[19] L. H. Peng, C. H. Ko, S. W. Siu et al., "In vitro \& in vivo assessment of a herbal formula used topically for bone fracture treatment," Journal of Ethnopharmacology, vol. 131, no. 2, pp. 282-289, 2010.

[20] Chinese Pharmacopoeia Commission, Pharmacopoeia of the People's Republic of China, Chinese Medical Science and Technology Press, Beijing, China, 2010.

[21] J. Li, X.-L. Wu, Y. Chen et al., "Antidiarrheal properties of different extracts of Chinese herbal medicine formula Bao-XieNing," Journal of Chinese Integrative Medicine, vol. 11, no. 2, pp. 125-134, 2013.

[22] G. Chen, K. K. Li, C. H. Fung et al., "Er-Miao-San, a traditional herbal formula containing Rhizoma Atractylodis and Cortex Phellodendri inhibits inflammatory mediators in LPSstimulated RAW264.7 macrophages through inhibition of NF$\kappa \mathrm{B}$ pathway and MAPKs activation," Journal of Ethnopharmacology, vol. 154, no. 3, pp. 711-718, 2014.

[23] R. Karki, C.-H. Park, and D.-W. Kim, "Extract of buckwheat sprouts scavenges oxidation and inhibits pro-inflammatory mediators in lipopolysaccharide-stimulated macrophages (RAW264.7)," Journal of Chinese Integrative Medicine, vol. 11, no. 4, pp. 246-252, 2013.

[24] Y.-X. J. Wang, C. H. Ko, J. F. Griffith et al., "Organic nitrate maintains bone marrow blood perfusion in ovariectomized female rats: a dynamic, contrast-enhanced magnetic resonance imaging (MRI) study," Pharmaceutics, vol. 5, no. 1, pp. 23-35, 2013.

[25] K.-W. Luo, J.-G. Sun, J. Y.-W. Chan et al., "Anticancer effects of imperatorin isolated from Angelica dahurica: induction of apoptosis in HepG2 cells through both death-receptor- and mitochondria-mediated pathways," Chemotherapy, vol. 57, no. 6, pp. 449-459, 2012.

[26] S. Wingerter, M. Tucci, J. Bumgardner, and H. Benghuzzi, "Evaluation of short-term healing following sustained delivery of osteoinductive agents in a rat femur drill defect model," Biomedical Sciences Instrumentation, vol. 43, pp. 188-193, 2007.

[27] A. Obenaus and P. Hayes, "Drill hole defects: induction, imaging, and analysis in the rodent," Methods in Molecular Biology, vol. 690, pp. 301-314, 2011.

[28] W. E. Hoffmann, N. Everds, M. Pignatello, and P. F. Solter, "Automated and semiautomated analysis of rat alkaline phosphatase isoenzymes," Toxicologic Pathology, vol. 22, no. 6, pp. 633-638, 1994.

[29] C. E. Wiedmeyer, D. G. Morton, P. K. Cusick, T. Wright, P. F. Solter, and W. E. Hoffmann, "Semiautomated analysis of alkaline phosphatase isoenzymes in serum of normal cynomolgus monkeys (Macaca fascicularis)," Veterinary Clinical Pathology, vol. 28, no. 1, pp. 2-7, 1999.

[30] S. B. Kessler, S. Deiler, M. Schiffl-Deiler, H. K. Uhthoff, and L. Schweiberer, "Refractures: a consequence of impaired local bone viability," Archives of Orthopaedic and Trauma Surgery, vol. 111, no. 2, pp. 96-101, 1992.

[31] S. Moncada, R. M. J. Palmer, and E. A. Higgs, "Nitric oxide: physiology, pathophysiology, and pharmacology," Pharmacological Reviews, vol. 43, no. 2, pp. 109-142, 1991. 
[32] D. O. Stichtenoth and J. C. Frölich, "Nitric oxide and inflammatory joint diseases," British Journal of Rheumatology, vol. 37, no. 3, pp. 246-257, 1998.

[33] M. B. Habal, "Controlled bone regeneration: the ultimate process in bone repair," Clinics in Plastic Surgery, vol. 29, no. 1, pp. 15-27, 2002.

[34] L. D. Esposti, L. Sinigaglia, M. Rossini et al., "Adherence to therapeutic and diagnostic recommendations in patients with femur fracture and at risk of re-fracture or death: results of an analysis of administrative databases," Reumatismo, vol. 64, no. 1, pp. 18-26, 2012.

[35] F. Shapiro, "Bone development and its relation to fracture repair. The role of mesenchymal osteoblasts and surface osteoblasts," European Cells and Materials, vol. 15, pp. 53-76, 2008.

[36] J. C. M. Teo, K. M. Si-Hoe, J. E. L. Keh, and S. H. Teoh, "Relationship between CT intensity, micro-architecture and mechanical properties of porcine vertebral cancellous bone," Clinical Biomechanics, vol. 21, no. 3, pp. 235-244, 2006.

[37] A. Syahrom, M. R. Abdul Kadir, J. Abdullah, and A. Öchsner, "Mechanical and microarchitectural analyses of cancellous bone through experiment and computer simulation," Medical and Biological Engineering and Computing, vol. 49, no. 12, pp. 1393-1403, 2011.

[38] L. Monfoulet, B. Rabier, O. Chassande, and J. C. Fricain, "Drilled hole defects in mouse femur as models of intramembranous cortical and cancellous bone regeneration," Calcified Tissue International, vol. 86, no. 1, pp. 72-81, 2010.

[39] H. Uusitalo, J. Rantakokko, M. Ahonen et al., "A metaphyseal defect model of the femur for studies of murine bone healing," Bone, vol. 28, no. 4, pp. 423-429, 2001.

[40] S. W. Veitch, S. C. Findlay, A. J. Hamer, A. Blumsohn, R. Eastell, and B. M. Ingle, "Changes in bone mass and bone turnover following tibial shaft fracture," Osteoporosis International, vol. 17, no. 3, pp. 364-372, 2006.

[41] B. L. Riggs and S. Khosla, "Role of biochemical markers in assessment of osteoporosis," Acta Orthopaedica Scandinavica, Supplementum, vol. 66, no. 266, pp. 14-18, 1995.

[42] P. Garnero, E. Hausherr, M.-C. Chapuy et al., "Markers of bone resorption predict hip fracture in elderly women: the EPIDOS Prospective Study," Journal of Bone and Mineral Research, vol. 11, no. 10, pp. 1531-1538, 1996.

[43] S. Vasikaran, R. Eastell, O. Bruyère et al., "Markers of bone turnover for the prediction of fracture risk and monitoring of osteoporosis treatment: a need for international reference standards," Osteoporosis International, vol. 22, no. 2, pp. 391420, 2011.

[44] K. Stoffel, H. Engler, M. Kuster, and W. Riesen, "Changes in biochemical markers after lower limb fractures," Clinical Chemistry, vol. 53, no. 1, pp. 131-134, 2007.

[45] P. Seebeck, H. J. Bail, C. Exner et al., "Do serological tissue turnover markers represent callus formation during fracture healing?" Bone, vol. 37, no. 5, pp. 669-677, 2005.

[46] C. Sousa, H. Abreu, C. Viegas et al., "Serum total and bone alkaline phosphatase and tartrate-resistant acid phosphatase activities for the assessment of bone fracture healing in dogs," Arquivo Brasileiro de Medicina Veterinaria e Zootecnia, vol. 63, no. 4, pp. 1007-1011, 2011.

[47] R. K. Schenk, "Biology of fracture repair," in Skeletal Trauma: Basic Science, Management, and Reconstruction, B. D. Browner, J. B. Jupiter, A. M. Levine, and P. G. Trafton, Eds., pp. 29-73, Saunders, Philadelphia, Pa, USA, 2003.
[48] J. Hadgraft and R. H. Guy, Transdermal Drug Delivery: Developmental Issues and Research Initiatives, Marcel Dekker, New York, NY, USA, 1989.

[49] G. E. Bast, "Influence of solubility and permeant size on absorption and metabolism of xenobiotics in rabbit skin," Human \& Experimental Toxicology, vol. 16, no. 8, pp. 435-440, 1997.

[50] R. K. Subedi, S. Y. Oh, M.-K. Chun, and H.-K. Choi, "Recent advances in transdermal drug delivery," Archives of Pharmacal Research, vol. 33, no. 3, pp. 339-351, 2010.

[51] M. J. Kruger and C. Smith, "Postcontusion polyphenol treatment alters inflammation and muscle regeneration," Medicine and Science in Sports and Exercise, vol. 44, no. 5, pp. 872-880, 2012. 


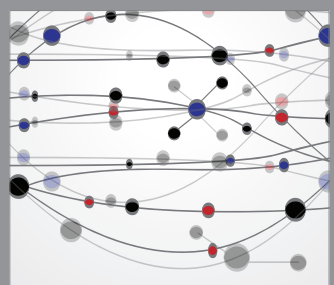

The Scientific World Journal
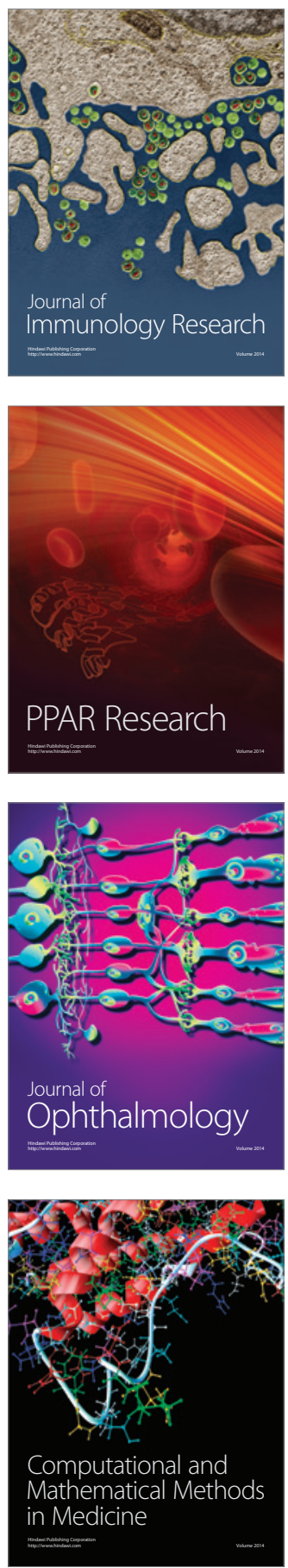

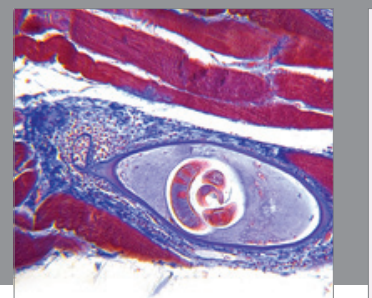

Gastroenterology

Research and Practice
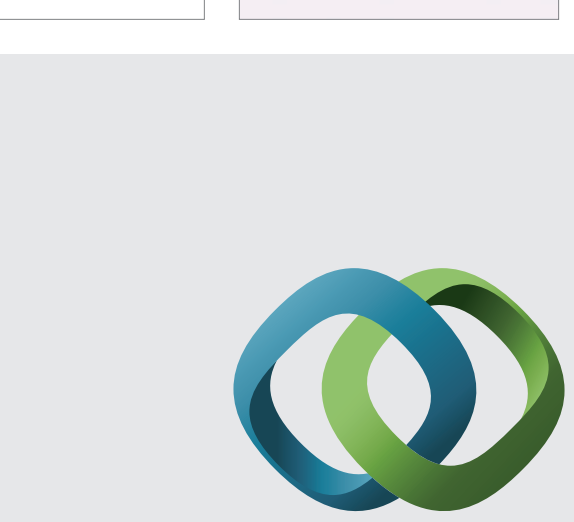

\section{Hindawi}

Submit your manuscripts at

http://www.hindawi.com
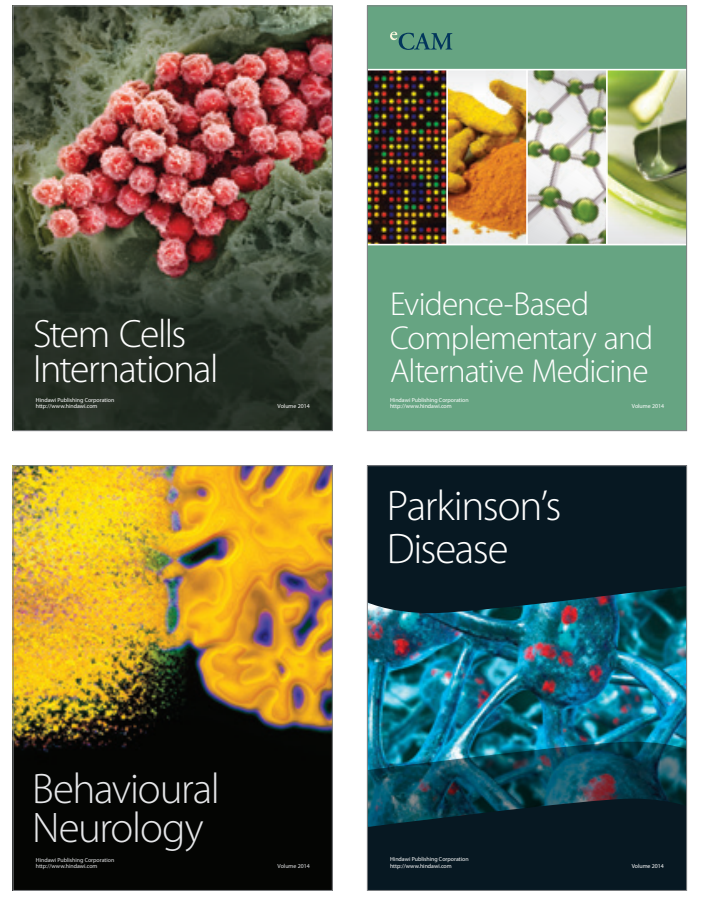
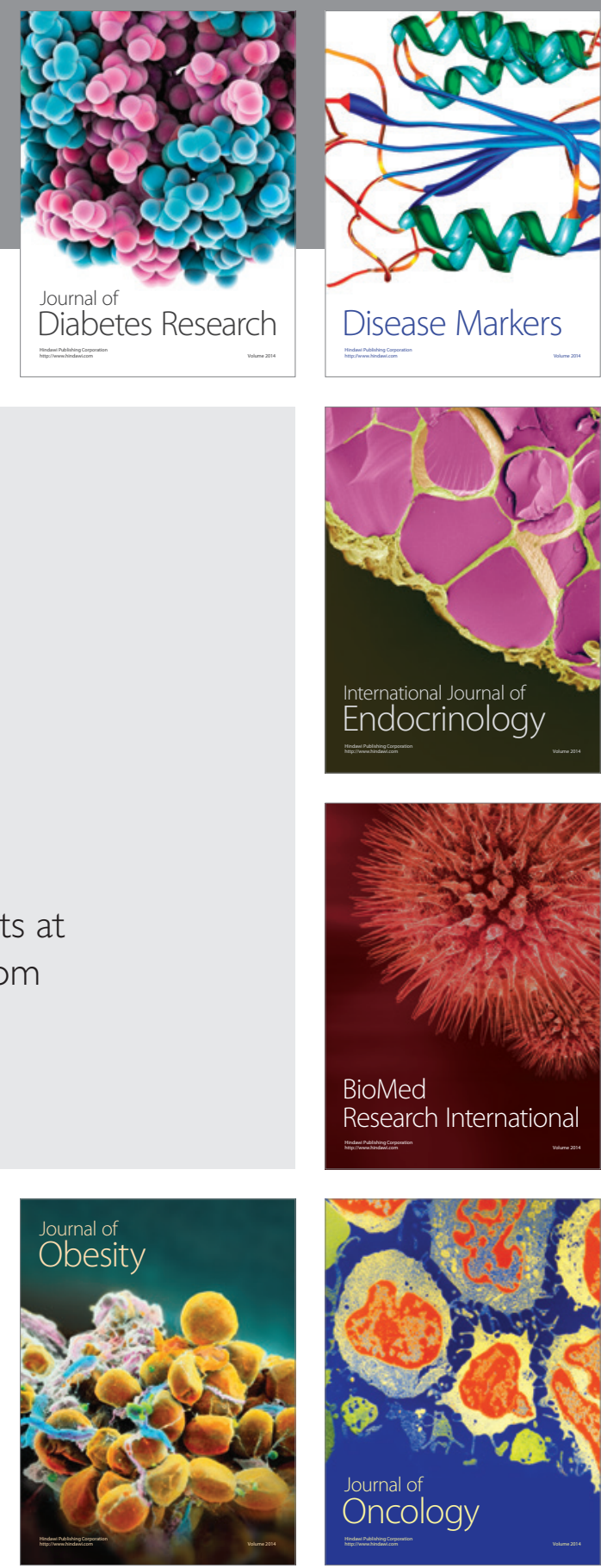

Disease Markers
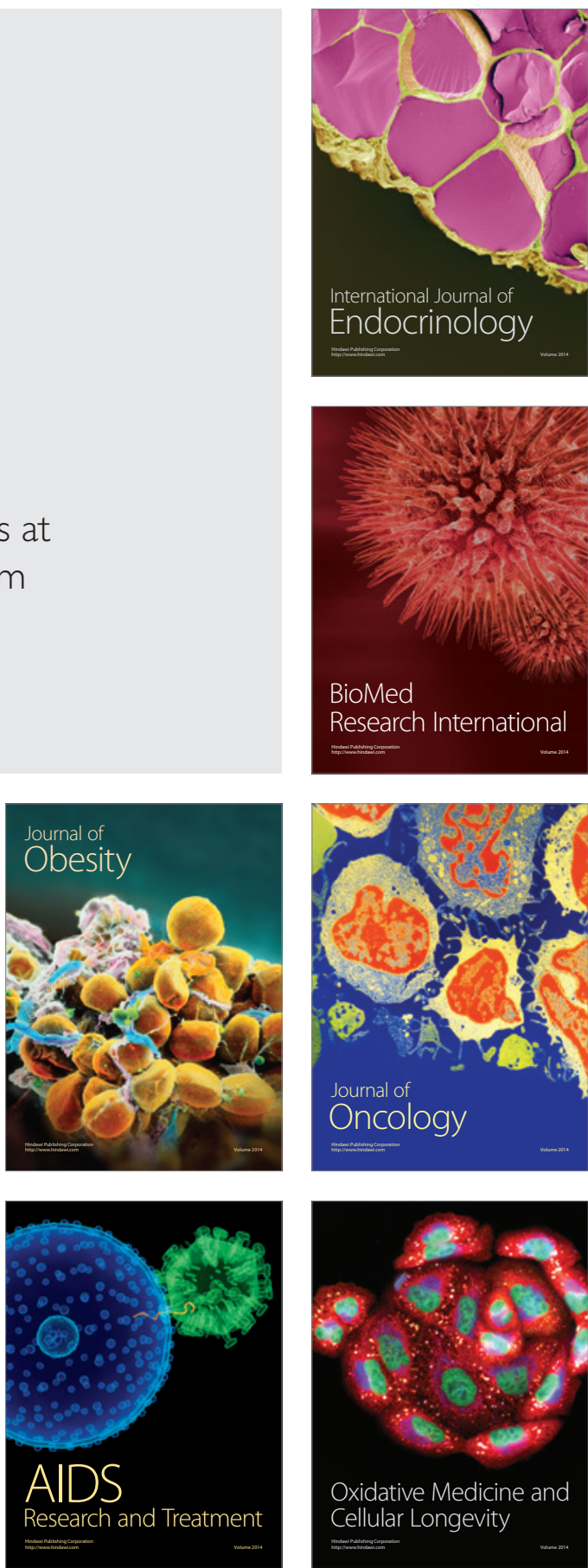